\title{
A Modular Approach to FEM-MOM Hybridization for the Analysis of Finite Arrays of Antennas
}

\author{
L. E. Garcia-Castillo ${ }^{1}$, Belen Andres ${ }^{1}$, I. Gomez Revuelto ${ }^{2}$, Luis E. Garcia-Muñoz ${ }^{1}$, Cristophe Craeye ${ }^{3}$ \\ ${ }^{1}$ Dep. Teoría de la Señal y Com., Universidad Carlos III de Madrid, Spain. luise@ît tsc.uc3m.es \\ Escuela Politécnica Superior, Avenida de la Universidad, 30, 28911. Leganés, Spain. [luise,belens,legarcia]@tsc.uc3m.es \\ ${ }^{2}$ Dpto. Ingeniería Audiovisual y Com. Universidad Politécnica de Madrid. E.U.I.T.T Ctra. Valencia Km. 7, 28031 Madrid. Spain \\ ${ }^{3}$ Université catholique de Louvain, Laboratoire TELE Place du Levant, 2, 1348 Louvain-la-Neuve. Belgium \\ christophe.craeye@uclouvain.be
}

\begin{abstract}
A modular approach is proposed to combine MoM and FEM for the fast analysis of finite arrays. The hybridization of MOM with FEM is obtained by using FEM to produce primary and secondary Macro Basis Functions (MBFs). A special mesh truncation technique based on domain decomposition is used. Once the MBFs are obtained, the finite array is solved via a reduced MOM problem with the MBFs so obtained as basis functions. The implementations of MOM+MBF and FEM methods are made in separate codes using surface triangular, and volumetric tetrahedral meshes, respectively
\end{abstract}

The Method of Moments is a very popular technique for the simulation of infinite and finite regular arrays of identical elements. Array elements containing both metallic and dielectric structures can be treated with the help of the surface equivalence, as done in [1]. An alternative for the analysis of arrays of complex elements containing dielectric media is the Finite Elements Method, which offers great flexibility in the treatment of complex structures, even in the case of nonhomogeneous dielectric objects.

In this paper, a modular approach is proposed to combine MoM and FEM for the fast analysis of finite arrays. The approach consists of defining equivalence surfaces on the boundaries of the elements of the array and to describe the equivalent electric and magnetic currents on those boundaries with the help of Macro Basis Functions (MBF). It is generally accepted that, as long as the the elements are small with respect to the wavelength, high accuracy can be achieved with relatively very few MBFs, which leads to a dramatic reduction of the number of unknowns when the elements of the array are complex. Besides this, the interactions between the MBFs can be computed very efficiently with the help of a spectral approach based on a Multipole decomposition of the freespace Green's function. In this case, in the intermediate-tofar field (for distances larger than about one wavelength), the interactions can be computed with a complexity that scales linearly with the area of the equivalence surface. For smaller distances, in regular arrays, very few relative distances need to be considered in the near field. Some of them are already considered upon computation of primary and secondary MBFs. An order of magnitude for the proportionality factor is obtained by knowing that for an object with about half-wavelength

The authors wants to acknowledge the support of Ministerio de Educacion y Ciencia of Spain under project TEC2007-65214/TCM. diameter, the number of MBFs may be limited to about 10 .

The hybridization of this approach with FEM is obtained by using the latter to produce primary MBFs and secondary MBFs (and potentially also higher orders) on the equivalence surface from the excitation of a unit cell either from inside (antenna mode) or from the outside incident field, as well as from fields produced in free space by other MBFs (scattering mode). With those products available, approaches like the on in [1] can be used to calculate the interactions between MBFs and, to solve the reduced system of equations and to obtain fields everywhere, including inside the equivalence surface. The FEM for scattering and radiation problems used is the one of [2], [3]. It is based on a non-standard iterative domain decomposition paradigm between the interior and exterior problems in order to provide an arbitrarily accurate (asymptotically exact with the number of iteration) radiation boundary condition while the original sparse and banded structure of the FEM matrices is retained. This mesh truncation methodology, referred to as FEIIEE (Finite Element - Iterative Integral Equation Evaluation, allows the FEM domain to be truncated very close to the sources of the problem reducing the number of unknowns.

The implementations of MOM+MBF and FE-IIEE methods are made in separate codes using surface triangular, and volumetric tetrahedral meshes, respectively. Furthermore, the former method uses first order $\mathbf{H}$ (div) basis functions (commonly known as RWG basis [4] in the EM literature) while the latter uses the second-order $\mathbf{H}$ (curl) basis of [5].

\section{REFERENCES}

[1] C. Craeye, T. Gilles, and X. Dardenne, "Efficient full-wave characterisation of anrays of antennas enbedded in finite dielectric volumes," Radio Science, vol. 44, Feb. 2009, doi:10.1029/2007RS003806.

[2] I. Gómez-Revuelto, L. E. García-Castillo, M. Salazar-Palma, and T. K. Sarkar, "Fully coupled hỵbrid method FEM/high-freguency technique for the analysis of radiation and scattering problems." Microwave and Optical Technology Letters, vol. 47, no. 2, pp. 104-107, Oct. 2005.

[3] R. Fernábdez-Recio, L. E. Garcia-Castillo, I. Gomez-Revuelto, and M. Salazar-Palma, "Fully coupled hybrid FEM-UTD method using NURBS for the analysis of radiation problems," IEEE Transactions on Antennas and Propagation, vol. 56, no. 3, pp. 774-783, Mar. 2008.

[4] S. M. Rao, D. R. Wilton, and A. W. Glisson, "Electromagnetic scattering by surfaces of arbitrary shape," IEEE Transactions on Antennas and Propagation, vol. 30, no. 3, pp. 409-418, May 1982.

[5] M. Salazar-Palma, T. K. Sarkar, L. E. García-Castillo, T. Roy, and A. R. Djordjevic, lterative and Self-Adaptive Finite-Elements in Electromagnetic Modeling. Norwood, MA: Artech House Publishers, Inc., 1998. 